Research Paper

\title{
Migration pattern, actin cytoskeleton organization and response to PI3K-, mTOR-, and Hsp90-inhibition of glioblastoma cells with different invasive capacities
}

\author{
Simon Memmel ${ }^{1}$, Dmitri Sisario ${ }^{1,2}$, Caren Zöller $^{1}$, Vanessa Fiedler ${ }^{1}$, Astrid Katzer ${ }^{1}$, \\ Robin Heiden ${ }^{1}$, Nicholas Becker ${ }^{1}$, Lorenz Eing ${ }^{2}$, Fábio L.R. Ferreira ${ }^{1}$, Heiko \\ Zimmermann $^{3,4}$, Markus Sauer ${ }^{2}$, Michael Flentje ${ }^{1}$, Vladimir L. Sukhorukov ${ }^{2, *}$ and \\ Cholpon S. Djuzenova ${ }^{1, *}$ \\ ${ }^{1}$ Department of Radiation Oncology, University Hospital of Würzburg, Würzburg, Germany \\ ${ }^{2}$ Department of Biotechnology and Biophysics, University of Würzburg, Würzburg, Germany \\ ${ }^{3}$ Fraunhofer-Institut für Biomedizinische Technik, Sulzbach, Germany \\ ${ }^{4}$ Molekulare und Zelluläre Biotechnologie/Nanotechnologie, Universität des Saarlandes, Saarbrücken, Germany \\ *Co-senior author \\ Correspondence to: Cholpon S. Djuzenova, email: djuzenova_t@ukw.de \\ Vladimir L. Sukhorukov, email: sukhoruk@biozentrum.uni-wuerzburg.de \\ Keywords: single-cell tracking, dSTORM, super-resolution microscopy, PTEN p53, wound healing \\ Received: January 12, $2017 \quad$ Accepted: March 20, $2017 \quad$ Published: April 05, 2017 \\ Copyright: Memmel et al. This is an open-access article distributed under the terms of the Creative Commons Attribution License 3.0 \\ (CC BY 3.0), which permits unrestricted use, distribution, and reproduction in any medium, provided the original author and source \\ are credited.
}

\section{ABSTRACT}

High invasiveness and resistance to chemo- and radiotherapy of glioblastoma multiforme (GBM) make it the most lethal brain tumor. Therefore, new treatment strategies for preventing migration and invasion of GBM cells are needed. Using two different migration assays, Western blotting, conventional and super-resolution (dSTORM) fluorescence microscopy we examine the effects of the dual PI3K/mTORinhibitor PI-103 alone and in combination with the Hsp90 inhibitor NVP-AUY922 and/or irradiation on the migration, expression of marker proteins, focal adhesions and F-actin cytoskeleton in two GBM cell lines (DK-MG and SNB19) markedly differing in their invasive capacity. Both lines were found to be strikingly different in morphology and migration behavior. The less invasive DK-MG cells maintained a polarized morphology and migrated in a directionally persistent manner, whereas the highly invasive SNB19 cells showed a multipolar morphology and migrated randomly. Interestingly, a single dose of $2 \mathrm{~Gy}$ accelerated wound closure in both cell lines without affecting their migration measured by single-cell tracking. PI-103 inhibited migration of DK-MG (p53 wt, PTEN wt) but not of SNB19 (p53 mut, PTEN mut) cells probably due to aberrant reactivation of the PI3K pathway in SNB19 cells treated with PI-103. In contrast, NVP-AUY922 exerted strong anti-migratory effects in both cell lines. Inhibition of cell migration was associated with massive morphological changes and reorganization of the actin cytoskeleton. Our results showed a cell linespecific response to PI3K/mTOR inhibition in terms of GBM cell motility. We conclude that anti-migratory agents warrant further preclinical investigation as potential therapeutics for treatment of GBM. 


\section{INTRODUCTION}

Glioblastoma multiforme (GBM) is a high-grade astrocytoma associated with a median survival time of 15 months, even after surgical resection, chemotherapy and radiotherapy [1]. Although more long-term survivors have been reported after combined chemo-radiotherapy, the high aggressiveness of GBM caused mainly by the diffuse infiltration of single tumor cells into the surrounding brain parenchyma makes complete tumor debulking virtually impossible $[2,3]$. Therefore, better therapies for GBM patients are necessary, involving strategies to prevent cell migration and invasion, which would enhance the tumor response to local treatment. These challenges have led to extensive efforts in elucidating the regulatory mechanisms of GBM cell motility in vitro and in vivo.

The mechanisms underlying GBM invasion in respect to molecular gene signatures are still unclear [4]. A typical glioblastoma harbors more than 60 genetic alterations [5]. The affected genes/pathways include, most notably, the dysregulated receptor tyrosine kinase (RTK), phosphatase and tensin homologue (PTEN), PI3K and inactivated p53 pathways. Frequent mutations in RTK, PTEN and $p 53$ genes contribute to a radio- and chemoresistant phenotype and also correlate with poor clinical outcome [6].

The tumor suppressor PTEN is deleted or mutated in $30 \%$ of GBMs and at lesser frequencies in other tumors [7]. PTEN inhibits cell migration, spreading, and focal adhesions, but its role in tumor invasion and metastasis is still unclear [8]. The second most commonly mutated gene in GBM is $p 53$ [5]. In literature, conflicting data exist concerning the impact of $p 53$ status on the resistance to cancer therapy [9]. Recent data indicate that some of the most common mutant p53 proteins have, in addition to losing transcriptional function, acquired a gain of function in promoting tumor cell migration and metastasis $[10,11]$. The effects of $\mathrm{p} 53$ on cell motility are largely mediated through the regulation of Rho signaling, thereby controlling actin cytoskeletal organization [12] and preventing filopodia formation, cell spreading, migration and invasion. Loss of p53 function increases the activities of RhoA and Rac through the activation of the PI3K/AKT/ mTOR signaling (hereafter denoted as the PI3K pathway), and also causes overabundance of $\mathrm{Cdc} 42$-dependent filopodia formation. As a result, activation of this network promotes cell adhesion and migration [13].

In addition to PTEN and p53, the activated PI3K pathway in PTEN-mutated cells can increase the migration and invasion activity of cells through either inhibition of 4E-BP1 (negative regulator of migration) or activation of S6K1 (positive regulator of migration) proteins [14]. Therefore, inhibition of key proteins in this pathway, such as PI3K, AKT and/or mTOR, can be expected to reduce the migration of tumor cells.

Besides this, tumor cell migration depends on the overexpression of the heat shock protein 90 (Hsp90). As a molecular chaperone, Hsp90 promotes the post- translational maturation and maintains the stability of a large number of oncogenic client proteins, including those implicated in cell migration [15].

Given the major roles of PI3K/mTOR and Hsp90 in regulating tumorcellmotility, weevaluated in this study whether their inhibitors, PI-103 and NVP-AUY922 (hereafter denoted as AUY922) have potential as anti-migratory agents in GBM. The novel synthetic molecule of the pyridofuropyrimidine class, PI-103, is a potent and selective inhibitor of class I PI3K [16], mTOR and DNA-PK, which exhibit therapeutic activity against a range of human tumor xenografts [17]. The second agent, NVP-AUY922, is an isoxazole resorcinol derivative with improved bioavailability, lower toxicity and high affinity for the $\mathrm{NH}_{2}$-terminal nucleotide-binding site of Hsp90 [18] along with beneficial pharmacological properties. It also exhibits strong antiproliferative effects against various tumor cell lines and primary tumors in vitro and in vivo at well-tolerated doses [19].

We analyzed the impact of either drug in combination with irradiation (IR) on the migration of two GBM cell lines differing in PTEN and p53 status. The cell lines were analyzed for cell morphology, F-actin distribution, migratory behavior in wound healing and single-cell tracking assays, and expression of several marker proteins of the PI3K and ERK pathways. Proteins responsible for cell adhesion and actin cytoskeleton organization (FAK, RhoA, Cdc42, etc.) were also examined.

\section{RESULTS}

\section{Migration patterns of GBM cells and effects of PI3K-, mTOR- and Hsp90-inhibition}

Using time-lapse video-microscopy we first examined the migration of individual DK-MG and SNB19 cells (Figure 1A, Supplementary Videos 1 and 2) using Time Lapse Analyzer. This software enables detailed analysis of individual cell tracks providing data on the migration speed and directionality (Figure 1D) of single cells.

As evident from the tracking diagrams of control samples shown in the upper row of Figure 1B, the final positions (black dots) of a large portion of DK-MG cells are more distant from the starting point than those of SNB19 cells. This finding suggests a higher directional persistence in the migration of control DK-MG cells as compared to a more random migration pattern of SNB19 cells. The latter conclusion is also supported by the statistical data shown in Figure $1 \mathrm{E}$ and $1 \mathrm{~F}$. Thus, although the migration speeds of control DK-MG and SNB19 cells are similar, the directionality index of DK-MG cells (Figure 1E) is significantly higher than that of control SNB19 cells (Figure 1F).

Besides different migration patterns, the two GBM lines also differ in the number of lamellipodia per cell (Figure 1C, Supplementary Videos 1 and 2). Thus, control DK-MG cells are strongly polarized in the direction of migration, exhibiting one large leading lamellipodium and 
a narrower trailing edge. Interestingly, at the leading edge of the lamellipodium, control DK-MG cells exhibit abundant transient blebs, visible in phase contrast microscopy as dark spherical expansions (Supplementary Video 1). Compared
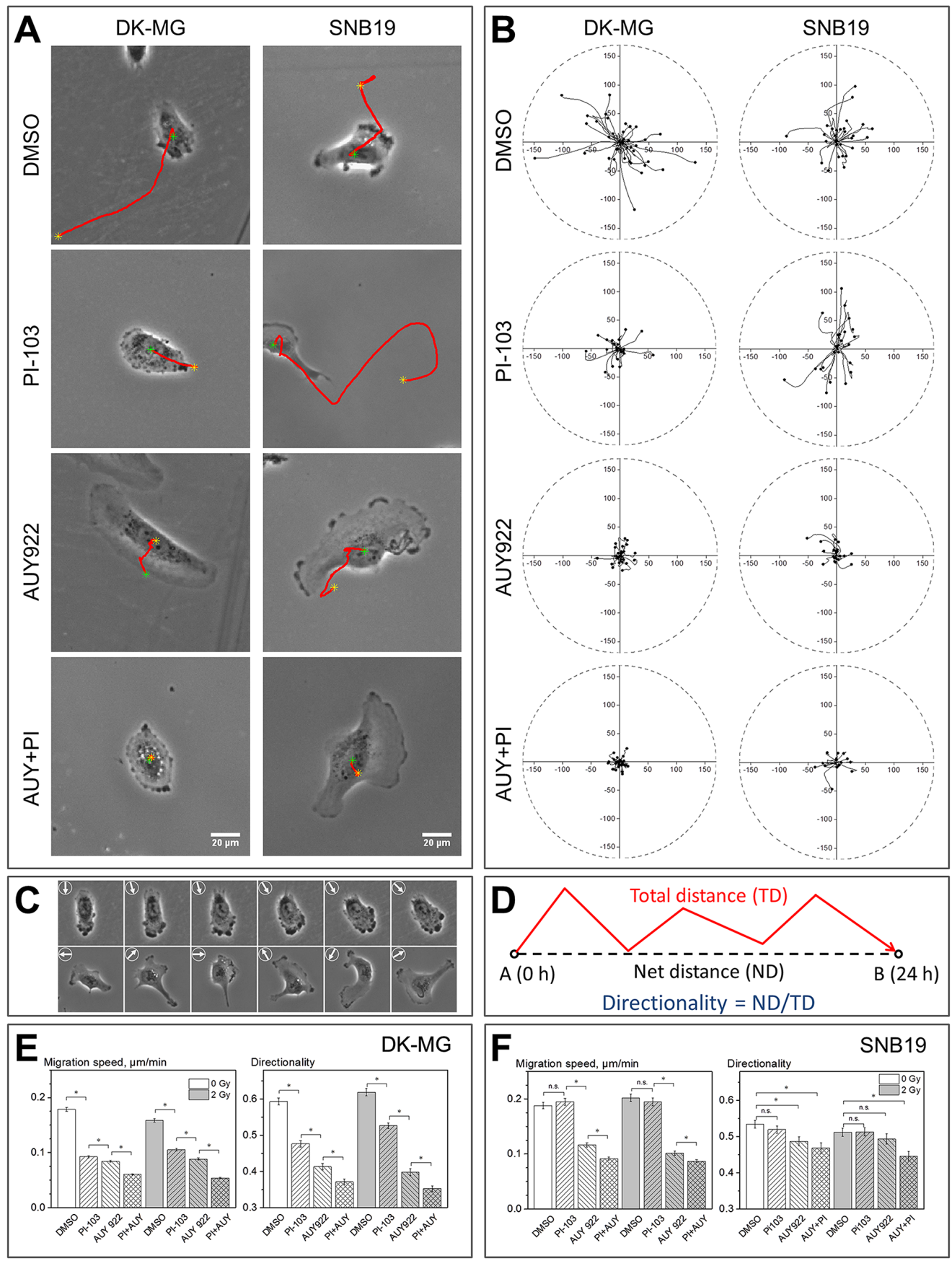

Figure 1: Impact of PI3K/mTOR and/or Hsp90 inhibition on the motility of DK-MG and SNB19 glioblastoma cell lines evaluated by single-cell tracking. (A) Typical appearance and migratory behavior of control (DMSO) and drug-treated cells observed by phase-contrast microscopy. Red lines indicate cell migration paths over a period of $5 \mathrm{~h}$. (B) Representative tracks of about 30 cells localized every 10 min for 5 hours, for each condition. The initial position of each cell was set to the origin $(0,0)$ of coordinates. Black dots indicate the final cell locations at time $=5 \mathrm{~h}$. The corresponding time lapse videos are available in Supplementary Videos 1 and 2. (C) Changes in cell morphology and direction of migration (arrows) for control DK-MG (upper row) and SNB19 (bottom row) cells. Images in (C) were taken in 100 min intervals over $500 \mathrm{~min}$. (D) The terms total and net distances, as well as the directionality of migration. Directionality of migration is defined as the net distance (ND, or displacement) from the starting position divided by the length of the total distance (TD). The results in (E) and (F) represent the mean values ( \pm SE) of migration speed (i.e. total distance divided by time) and directionality (D) from at least 500 cells per condition. White and grey bars denote non-irradiated and irradiated (2 Gy) samples, respectively. * means $P<0.05$. "n.s." means "not significant". 
to control DK-MG cells, the random migration pattern of control SNB19 cells (Supplementary Video 2 and arrows in Figure 1C) might have resulted from uncoordinated activity of multiple lamellipodia formed by this cell line, exhibiting multipolar morphology and fast morphological changes (Figure 1C, Supplementary Video 2).

Treatment with either PI-103 or AUY922 decreased the migration speed of DK-MG cells by $\sim 50 \%$, with respect to the DMSO-treated control (Figure 1E). Simultaneous treatment with PI-103 and AUY922 gave rise to a much stronger (67\%) inhibition of DK-MG cell migration (Figure 1E). In sharp contrast to its strong effect on DK-MG cells, PI-103 alone did not affect the migration of SNB19 cells (Figure 1F). On the other hand, AUY922 given either alone or in combination with PI103 significantly reduced the migration speed of nonirradiated SNB19 cells (Figure 1F), although to a lesser extent than in DK-MG cells (Figure 1E). In both cell lines, combination of PI-103 and AUY922 caused a stronger reduction in migration speed than either inhibitor alone (Figure 1E and 1F).

Irradiation with 2 Gy (grey bars in Figure 1E and $1 F$ ) caused little, if any, changes in the migration activities of single cells from both cell lines, as compared to the corresponding non-irradiated samples (white bars). Likewise, the effects of PI-103 and AUY922 on the migration of irradiated cells were similar to their effects on non-irradiated samples from both cell lines.

In addition to single-cell tracking, we performed a wound healing assay. In agreement with the results of the single-cell tracking assay, drug-treated DK-MG cells closed the wound area much slower than control (i.e. drugfree and non-irradiated) cells (Figure 2A). The data of the wound healing test on both cell lines, are statistically summarized in Figure 2B and 2C. Treatment with PI-103, AUY922, or both agents combined almost completely
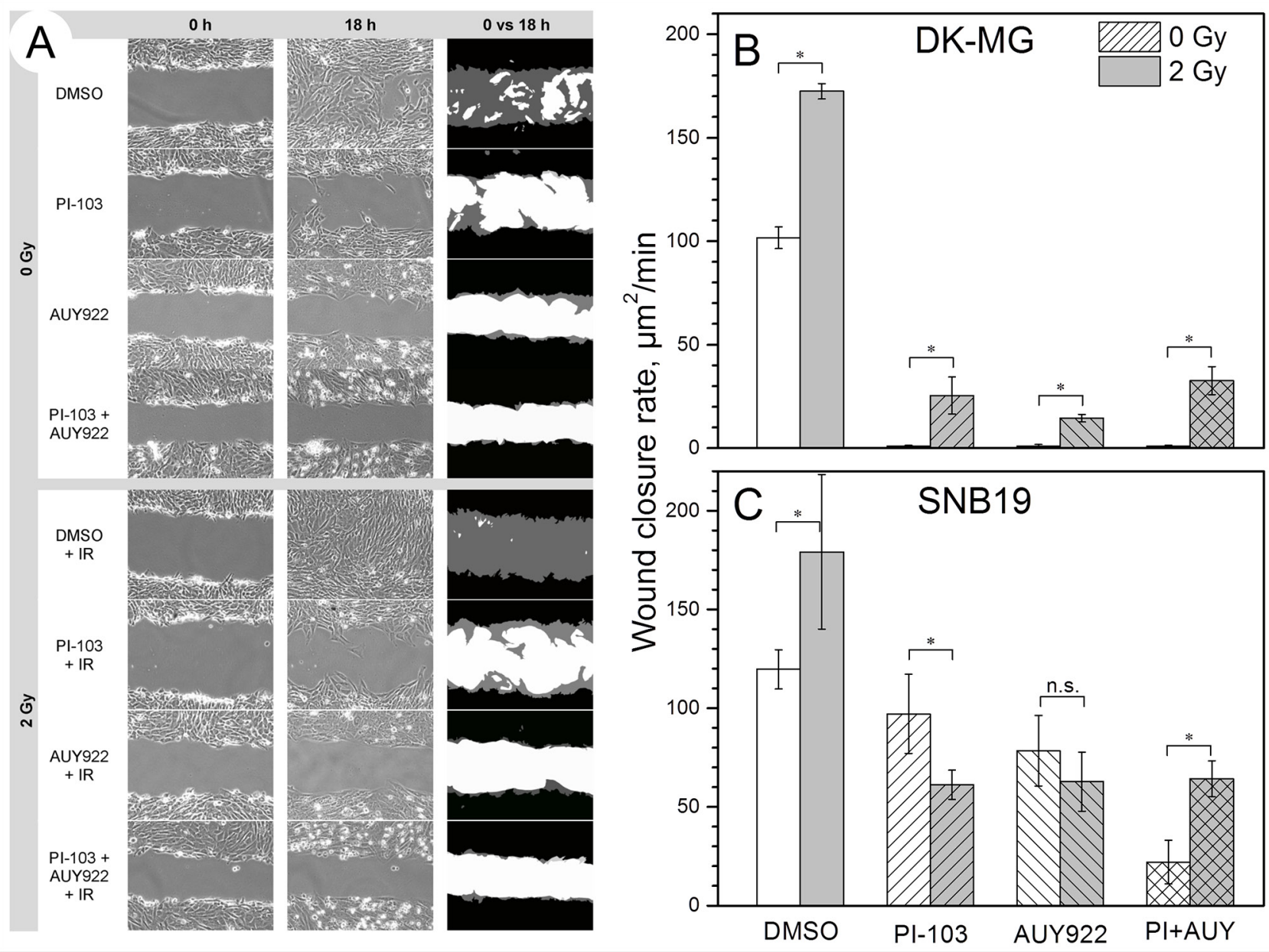

Figure 2: Effects of PI3K/mTOR and Hsp90 inhibition on the migration of non-irradiated and irradiated (2 Gy) cells studied by a wound healing assay. Representative phase contrast images of DK-MG cells shown in the left and middle columns in (A) were acquired at 0 and $18 \mathrm{~h}$ intervals, respectively, after the scratch was made. White color in the RHS column of (A) represents the cell-free area, black and grey colors denote cell-covered areas at time $=0$ and $18 \mathrm{~h}$, respectively. The bar graphs in (B) and (C) summarize the impact of drug treatment on the wound closure rate, expressed in unit area per min $\left[\mu \mathrm{m}^{2} / \mathrm{min}\right]$, in DK-MG (B) and SNB19 (C) cell monolayers. Each bar represents the mean $\pm \mathrm{SE}$ of at least three independent experiments made in quadruplicate. 
inhibited migration of DK-MG cells into the wound (Figure 2A).

A further finding of the wound healing test was that SNB19 cells were less responsive to drug treatment than DK-MG cells (Figure 2C). Thus, compared to untreated controls, SNB19 cells treated with PI-103, AUY922, and both agents combined, exhibited, respectively, a 19, 34 and $82 \%$ decrease in the wound healing rate (Figure $2 \mathrm{C}$ ), which is much less than the 100-fold inhibition observed in drug-treated DK-MG cells (Figure 2B). Interestingly, a single IR dose of 2 Gy accelerated the wound closure rate in control and drug-treated samples of both GBM lines (grey bars in Figures 2B and 2C).

\section{Effects of PI3K-, mTOR- and Hsp90-inhibition on the actin cytoskeleton}

Cell migration is a complex process that involves coordinated changes in the F-actin cytoskeleton and focal adhesions [20]. In order to explain the different response of two GBM lines to a dual PI3K/mTOR inhibitor in terms of cell migration (Figures 1 and 2), we analyzed, via fluorescence microscopy, the drug-induced remodeling of actin filaments and focal adhesion complexes.

Confocal laser scanning microscopy (LSM) demonstrated substantial differences between control DK-MG and SNB19 cells in the organization of the actin cytoskeleton and focal adhesions (Figure 3A and 3B). Combined staining of DK-MG cells for F-actin (magenta) and focal adhesion kinase phosphorylated at tyrosine 397 (p-FAKTyr397, green) clearly shows their polarized shape (i.e. the front-rear polarity) along with different types of actin stress fibers, including dorsal fibers and transverse arcs, typical for the leading edge of the lamellipodium [21]. In addition to conventional LSM, the lamellipodial actin meshwork of control DK-MG cell was resolved in more detail by means of super-resolution imaging using direct stochastic optical reconstruction microscopy (dSTORM) [22, 23], as shown in the two bottom pictures in the left-hand side (LHS) column of Figure 3A. While being evenly distributed through the cytosol of control DK-MG cells (top left image of Figure 3A), p-FAK is highly concentrated at the leading edge and, to a lesser extent, at the cell rear, where it co-localizes with F-actin within the focal adhesions (Figure 3A, inset in top left image).

In contrast to DK-MG cells, control SNB19 cells analyzed by LSM show multipolar morphology with several lamellipodia protruding from the cell body in all directions (top left image in Figure 3B), which agrees well with the phase-contrast images (Figure 1C). The tip of each lamellipodium shows diffuse actin staining along with p-FAK-enriched spots in the focal adhesions (Figure $3 \mathrm{~B}$, inset in top left image). dSTORM images (Figure $3 \mathrm{~B}$, bottom left image) confirm diffuse actin staining, resulting most likely from the gel-like actin structure in the lamellipodial tips. Unlike unipolar DK-MG cells, multipolar SNB19 cells contain very few, if any, stress fibers and focal adhesions in the central regions.

Irrespective of the drug used, the most notable change in drug-treated DK-MG cells is the lack of a polarized cytoskeleton structure (Figure 3A) and of the transverse arcs seen in migrating control cells. Besides this, both LSM and $d$ STORM reveal prominent actin stress fibers anchored to focal adhesions at the perimeter (Figure 3A) of PI-103-treated DK-MG cells. The central region of these cells contains neither ventral stress fibers nor focal adhesions detectable by LSM. However, taking advantage of super-resolution $d$ STORM we found tiny, randomly oriented actin fibers with a diameter of 70-200 $\mathrm{nm}$ (i.e. beyond the resolution limits of LSM) close to the ventral plasma membrane. AUY922-treated DK-MG cells display abundant radial stress fibers terminated with focal adhesions at the cell periphery (Figure 3A). Although PI103 did not affect the migration of SNB19 cells in the single-cell tracking test (Figure 1F) or exhibited at best modest efficacy in the wound healing test (Figure 2C), it caused a reorganization of actin filaments, most notably the loss of multiple lamellipodia, seemingly due to them merging into one large lamellipodium. The actin-rich leading edge of this lamellipodium contains abundant focal adhesions, whereas the cell rear is largely devoid of F-actin and focal adhesions.

Along with strongly impeded migration, SNB19 cells treated with AUY922 exhibit a marked enlargement of the projected cell area, numerous actin stress fibers and focal adhesions in the central regions, as well as dense actin staining and focal adhesions along the entire cell border (Figure 3B), which is consistent with our previous results obtained by epifluorescence microscopy [24]. In both cell lines, a single radiation dose of 2 Gy applied alone or in combination with either inhibitor did not cause any significant changes in the actin cytoskeleton, as compared to the corresponding non-irradiated samples (data not shown).

\section{Effects of PI3K-, mTOR- and Hsp90-inhibition on marker protein expression}

The data presented in Figures 1-3 demonstrate the ability of the PI3K/mTOR/Hsp90 inhibitors to modify the morphology of GBM cells and, most notably, to inhibit their migration, in a cell line-specific manner. To gain an insight into the mechanisms responsible for the observed differences between the two cell lines, we examined by Western blot the expression of two groups of marker proteins. The first group of proteins (Figure 4A) includes targets of the dual inhibitor PI-103, i.e. PI3K and mTOR, along with p-AKT and p-4E-BP1. Because of the known crosstalk between PI3K and ERK pathways [25], proteins of the ERK signaling pathway (Raf-1, p-MEK1/2 and p-ERK1/2) were also tested. The ERK pathway, which is 
A
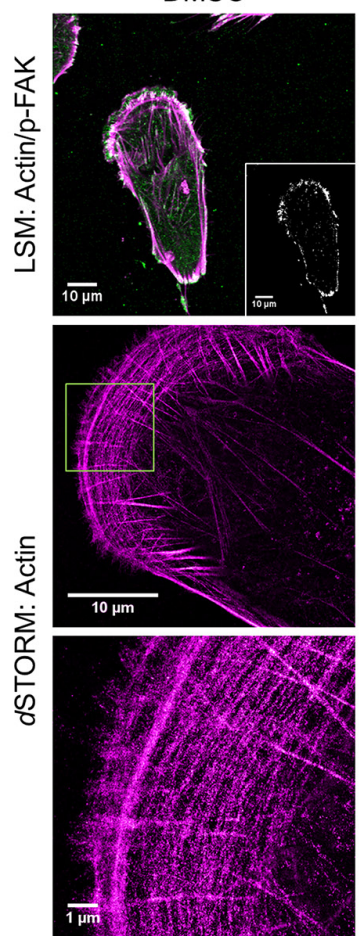

B
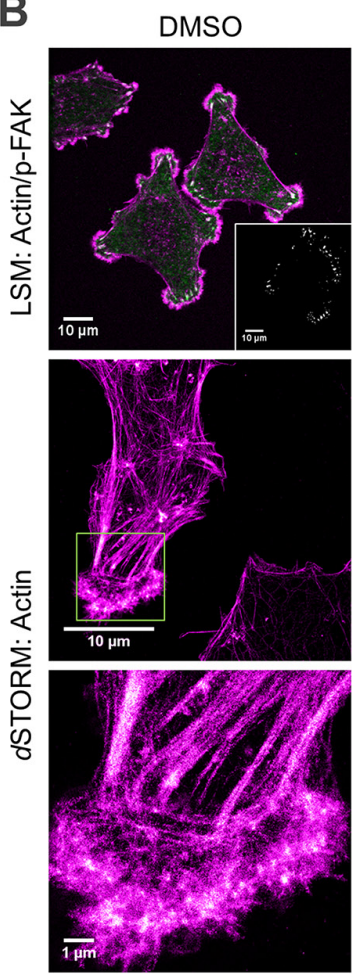

PI-103
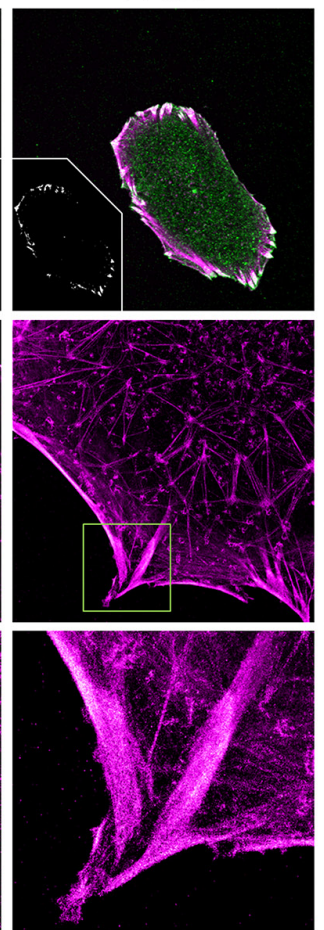

Pl-103
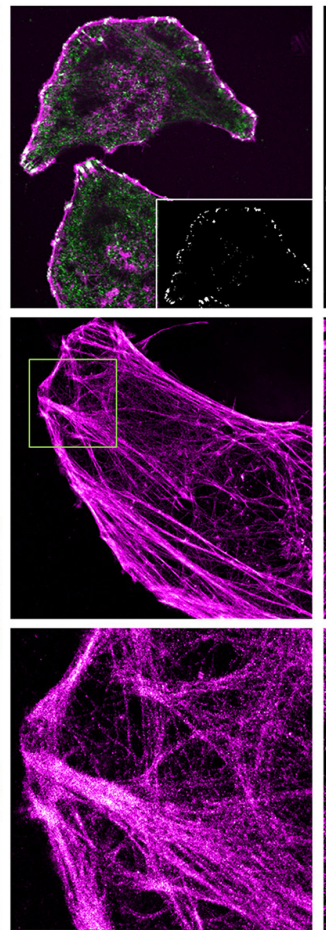

AUY922
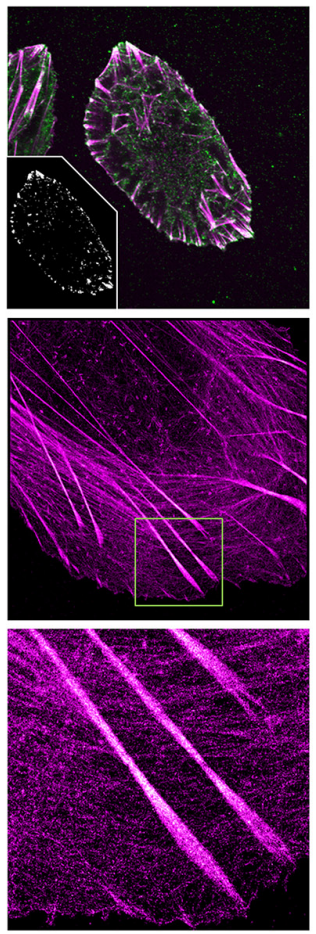

AUY922
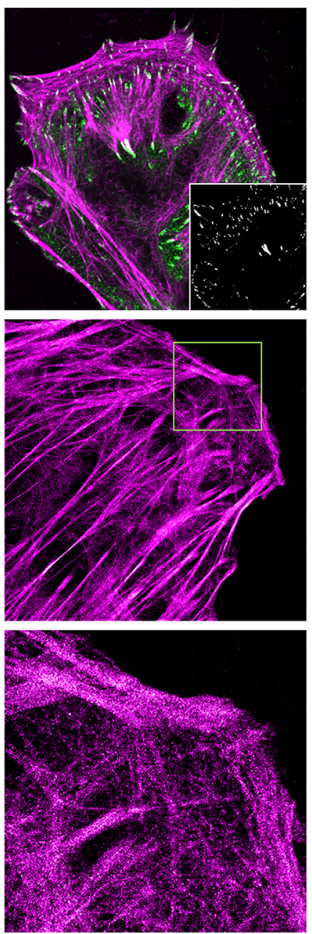

Figure 3: Effects of PI-103 and AUY922 on F-actin organization (magenta) and distribution of p-FAK(Tyr397) (green, upper row) in DK-MG (A) and SNB19 (B) cells. Images were acquired by confocal (LSM, upper row) and super-resolution ( $d$ STORM, middle and bottom rows) microscopy. Images show cell sections close to the basal plasma membrane in order to visualize focal adhesions and actin structure. Insets in the upper row illustrate co-localization of actin with p-FAK(Tyr397) (white area) at focal adhesion sites. The bottom row shows a magnification of the areas outlined by yellow squares in the corresponding $d$ STORM images (middle row). F-actin filaments were visualized with Dy647-conjugated phalloidin. p-FAK(Tyr397) was immunostained with primary and secondary antibodies labeled with Alexa488 (see Supplementary Materials). Scale bars are $10 \mu \mathrm{m}$ in the upper and middle rows and $1 \mu \mathrm{m}$ in the bottom row, respectively. 
frequently mutated in cancer cells [26], transmits signals from surface receptors to promote survival, proliferation and migration [27].

The second group of proteins (Figure 4B) includes the Rho GTPases, namely RhoA, Cdc42, and Rac1/2/3, which require phosphorylation by pro-survival signaling through AKT and ERK2 [28]. The members of the Rho GTPase family regulate intracellular actin dynamics, cytoskeleton remodeling, cell polarity and lamellipodium extension [29-32]. In addition, we detected
p-FAK(Ser910). Representative Western blots of the indicated proteins are shown in Figure 4 for control (DMSO) and drug-treated (24 h) DK-MG and SNB19 (LHS and right-hand (RHS) columns of the figure, respectively) cells. The data on short-term $(3 \mathrm{~h})$ treatment are shown in Supplementary Figure 1.

As seen in Figure 4A, control DK-MG and SNB19 cells contain comparable amounts of PI3K, p-mTOR and Raf-1. At the same time, expression of $\mathrm{p}-\mathrm{AKT}$, p-MEK1/2 and p-ERK1/2 in control SNB19 cells is much

DK-MG

SNB19

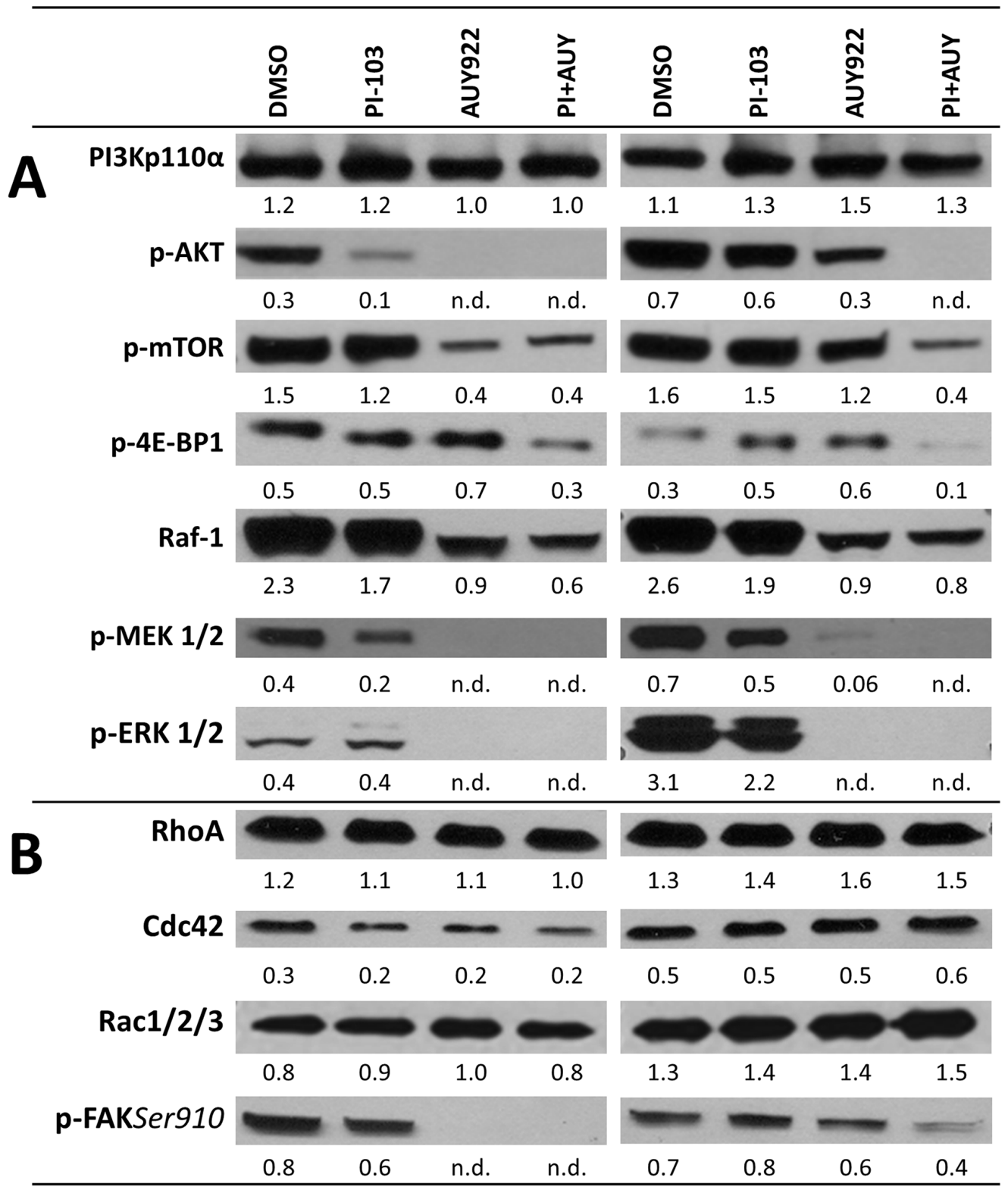

Figure 4: Representative Western blots of several marker proteins of PI3K- and ERK-pathways (A), the Rho GTPases (RhoA, Cdc42 and Rac 1/2/3) and p-FAK(Ser910) (B) in DK-MG and SNB19 cells treated with DMSO (control) or the indicated inhibitors for 24 h. Each protein band was normalized to the intensity of $\beta$-actin used as loading control. The protein $/ \beta$-actin ratios are denoted by the numbers. The experiments were repeated at least three times. 
higher than in control DK-MG cells. The increased level of p-AKT (Figure 4A) in PTEN-mutated SNB19 cells (Supplementary Figure 2) is consistent with the findings that PTEN loss can lead to compensatory activation of the PI3K pathway [33]. The amount of p-4E-BP1 in DK-MG cells exceeds that of SNB19 cells. The level of p-FAK $(\operatorname{Ser} 910)$ in control SNB19 cells is $25 \%$ lower than in control DK-MG cells (Figure 4B). At the same time, SNB19 cells show higher (by 20-50\%) expression levels of the Rho GTPases (RhoA, Cdc42 and Rac1/2/3), which might be associated with the $p 53$ mutation in SNB19 cells, since loss of p53 function activates RhoA and Rac, and also causes overabundance of $\mathrm{Cdc} 42$-dependent filopodia formation [13].

Prolonged incubation with PI-103 caused a significant decrease in p-AKT, p-MEK1/2, Raf- 1 and p-mTOR in DKMG cells, but it had little, if any, impact on these proteins in SNB19 cells. Interestingly, PI-103 increased the expression of the phosphorylated form of 4E-BP1 in SNB19 cells which can result in an activation of overall translation and migration, whereas its content was almost unchanged in PI-103-treated DK-MG cells. Besides this, PI-103 strongly decreased the expression of $\mathrm{Cdc} 42$ and p-FAK $(\operatorname{Ser} 910)$ in DK-MG cells, without significantly affecting RhoA and Rac1/2/3. In PI-103-treated SNB19 cells, there was a slight increase in Rho GTPase and p-FAK(Ser910) levels, with respect to DMSO-treated control. As expected, treatment with AUY922 strongly increased the expression of Hsp90 (Supplementary Figure 3) but decreased the expression of Hsp90 client proteins [15], e.g. p-AKT, p-MEK1/2, and p-ERK $1 / 2$, in both cell lines. Interestingly, the expression of p-AKT was almost completely abolished in AUY922treated DK-MG cells whereas it was decreased only by a factor of $\sim 2$ in the respective SNB19 cell samples. This may be due to the large difference in $\mathrm{p}-\mathrm{AKT}$ expression levels between the control samples of the two cell lines. Determination of the non-phosphorylated forms of the marker proteins (Supplementary Figures 4 and 5) revealed that their expression was less affected by either inhibitor as compared to the respective phosphorylated forms of the proteins (Figure 4).

\section{DISCUSSION}

In this study, we tested the effects of targeting the PI3K pathway using the dual PI3K/mTOR inhibitor PI103 with the intention of preventing the migration of two GBM cell lines differing in p53 and PTEN status [34] as well as in their invasive capacities [35]. As an alternative approach, we evaluated the anti-migratory potential of the Hsp90 inhibitor AUY922. Briefly, we found that $(a)$ the two GBM lines exhibited very different migratory patterns, i.e. directionally persistent $v$ s. random movement; $(b)$ inhibition of $\mathrm{PI} 3 \mathrm{~K} / \mathrm{mTOR}$ greatly reduced migration of the less invasive DK-MG cells (PTEN wt, $p 53 \mathrm{wt}$ ), without affecting migration of the highly invasive
SNB19 cells (PTEN mut, p53 mut); (c) in contrast, the Hsp90 inhibitor AUY922 exerted in both cell lines strong anti-migratory effects, which were further enhanced by the addition of PI-103 (Figures 1 and 2).

The most striking difference between the two GBM lines, revealed by single-cell tracking, is that DK-MG cells move persistently in one direction, whereas SNB19 cells exhibit a more random migration (Supplementary Videos 1 and 2). A further difference is that only DK-MG cells, but not SNB19 cells, form abundant membrane blebs at the leading edge of the lamellipodium (Supplementary Video 1). Such membrane blebbing has also been observed in various other cell types during migration, both in vitro and in vivo [36].

An interesting finding is the significant increase in the wound closure rates observed in both cell lines after exposure to IR (Figure 2). Radiation-induced acceleration of GBM cell migration has already been reported [37]. This effect could be due to IR-induced release of exosomes, which has been shown to enhance GBM cell migration [38]. The abundance of exosomes in multicellular systems (such as a confluent cell monolayer) would explain why IR led to increased cell migration in the wound healing assay (Figure 2), but not in the singlecell tracking test (Figure 1).

The different migration patterns of the two cell lines apparently result from the cell line-specific expression profiles of relevant proteins (Figure 4), which in turn might be associated with the PTEN (and possibly p53) mutation status of these cell lines. The observed differences in cell migration patterns between the two cell lines are explained by a simplified model presented in Figures 5A and 5B.

In general, our Western blot detections (Figure 4) showed higher activities of the key components of the PI3K and ERK pathways in PTEN-mutated SNB19 cells, as compared to the PTEN wild-type DK-MG cells. Both PI3K and ERK pathways include critical factors not only for enhanced proliferation and survival, but also for the promotion of migratory and invasion machineries [31, 39-41].

In addition to PTEN, cell motility is controlled by p53 [13], present as wild-type in DK-MG, but mutated in SNB19 cells. A deficiency in p53 has been shown to be implicated in the development of highly aggressive tumors [42]. As reviewed elsewhere [13], loss of p53 function promotes (via activation of PI3K pathway) upregulation of Rho GTPases, which is consistent with the results reported here showing higher Rho GTPase (RhoA, Cdc42, and Rac) levels in p53-mutated SNB19 cells as compared to p53 wild-type DK-MG cells (Figure 4B).

Along with the differences between control cells of the two GBM lines, the most important finding of this study, confirmed by two independent migration assays (Figures 1 and 2), is that PI3K/mTOR inhibition by PI103 exerted a strong anti-migratory effect only on DKMG but not on SNB19 cells. The potential of PI-103 as 
A

Control DK-MG cells

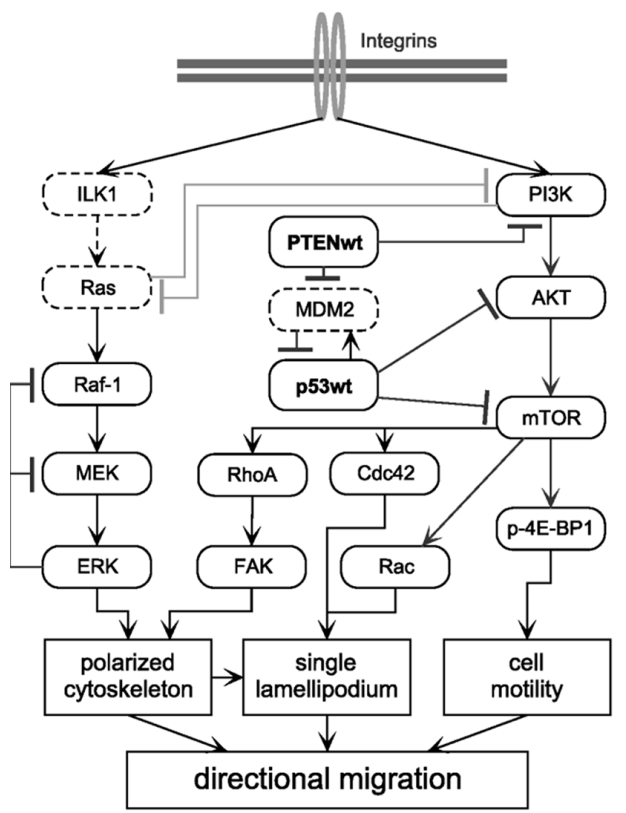

C

DK-MG cells + PI-103

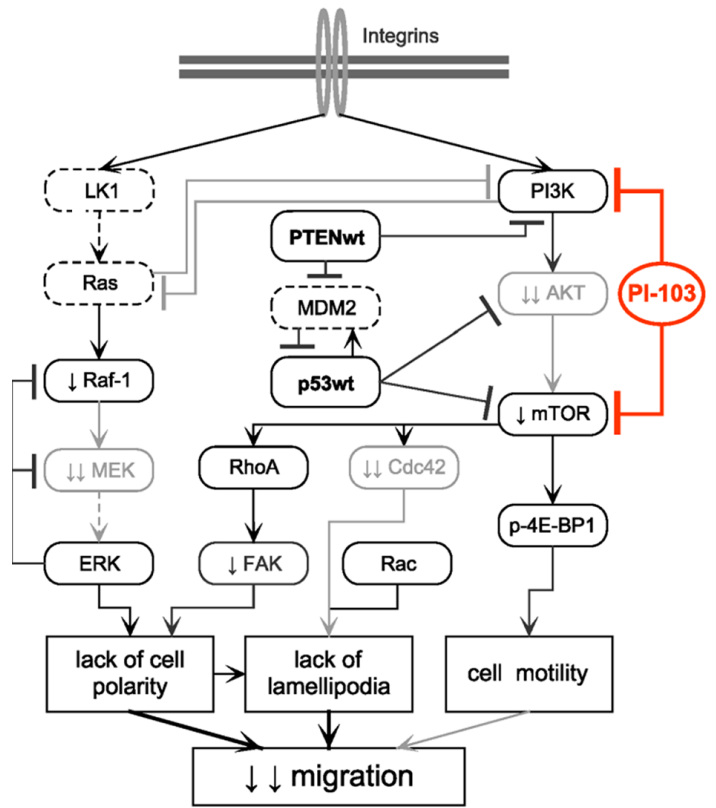

B

Control SNB19 cells

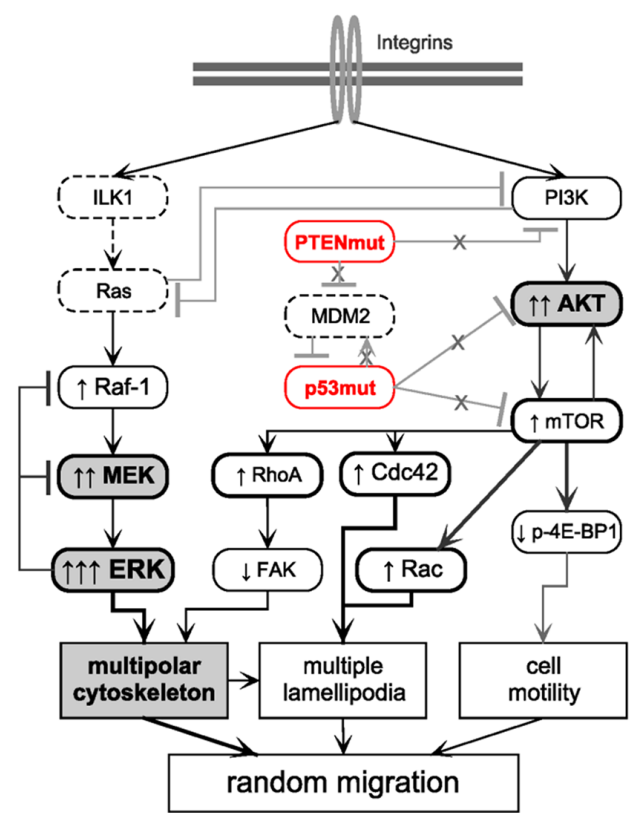

D

SNB19 cells $+\mathrm{PI}-103$

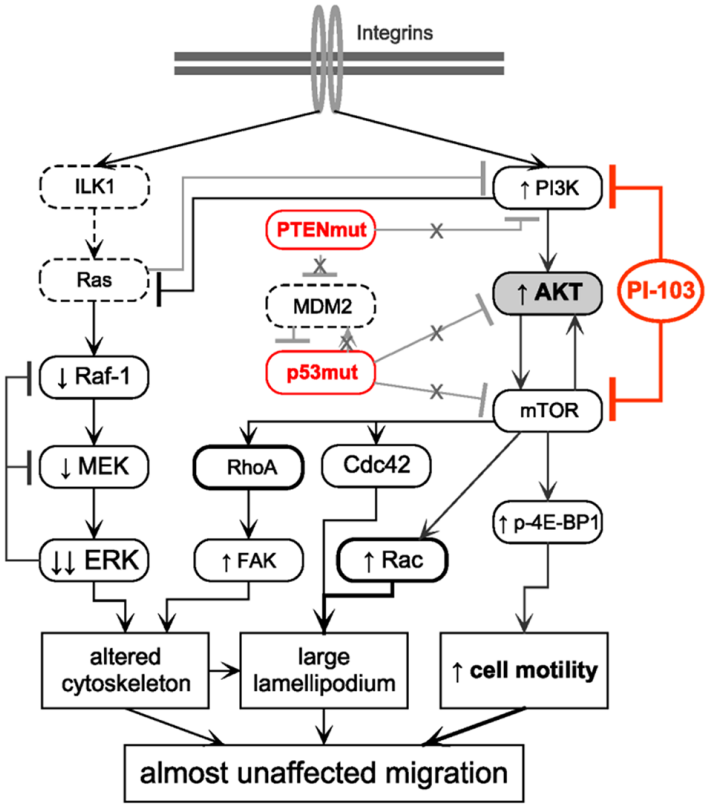

Figure 5: Simplified diagrams of putative signaling pathways responsible for the different migration patterns (i.e. directionally persistent vs. random) of control DK-MG (A) and SNB19 (B) cells, as well as for the cell line-specific effect of the PI3K/mTOR inhibitor PI-103 on cell migration (C) and (D). In contrast to DK-MG cells, the PTEN-mutated SNB19 cells express constitutively activated PI3K- and ERK-pathways which favor migration and invasion of these cells. Besides this, the protein p53 is mutated in SNB19 cells, which might also influence their migration phenotype, e.g. increased expression of RhoA, Cdc42 and Rac1/2/3. Incubation of SNB19 cells with PI103 for $24 \mathrm{~h}$ (D) leads to a rebound activation of the PI3K/AKT/mTOR pathway, most likely due to inhibition of the negative feedback loop mediated by ribosomal protein S6, resulting in mostly unaltered motility. In contrast, in PI-103-treated DK-MG cells, which lost their motility, the expression of p-AKT remained almost undetectable. The diagram is based on the data presented in Figures 1-4 and Supplementary Videos 1 and 2, and also includes data published elsewhere [25, 26, 29, 30]. Non-determined proteins are marked with dashed lines. The symbols $\uparrow$ and $\downarrow$ denote, respectively, increased and decreased protein expression levels compared to corresponding control cells (A, B), as detected by Western blot analysis shown in Figure 4. (For detail, see the Discussion section.) 
an inhibitor of cell migration has been tested by Ströbele et al. [43], who report that PI-103 reduces cell motility in two established and three primary GBM cell lines.

Cell line-specific effects of PI-103 (Figures 1 and 2) are also evident on the protein expression level (Figure 4). Particularly, PI-103 has a strong inhibitory effect on the expression of p-AKT, p-MEK1/2, p-FAK(Ser910) and Cdc42 only in DK-MG cells. Based on our results of the migration tests (Figures 1 and 2), fluorescence microscopy of the cytoskeleton (Figure $3 \mathrm{~A}$ and $3 \mathrm{~B}$ ) and Western blot analysis (Figure 4), we propose a simplified model explaining the anti-migratory effect of PI-103 on DK-MG cells and the lack of effect on SNB19 cells (Figure 5C and 5D).

A possible reason for the failure of PI-103 to inhibit migration of SNB19 cells might be rebound activation of AKT in this cell line upon 24-h drug exposure (Figures $4 \mathrm{~A}$ and 5D), which occurred after a transient p-AKT depletion observed in cells treated with PI-103 for $3 \mathrm{~h}$ (Supplementary Figure 1). In contrast, PI-103-treated DKMG cells remained almost depleted of p-AKT (Figures $4 \mathrm{~A}$ and $5 \mathrm{C}$ ) during the whole incubation period. We hypothesize that reactivated AKT and the signaling events downstream of it may contribute (via a yet unknown mechanism) to the unaffected motility of PI-103-treated SNB19 cells. One explanation is that activated AKT is known to promote cell motility downstream of Rac/Cdc42 GTPases thereby modulating the cytoskeleton in growth factor-stimulated cells and in invasive PTEN-deficient cells [44], such as the SNB19 cells studied here.

In addition, given that both activated FAK and Cdc42 are important for maintaining a polarized cytoskeleton and for forming membrane protrusions [45], the observed reduction of p-FAK $(\operatorname{Ser} 910)$ and $\mathrm{Cdc} 42$ in PI-103-treated DK-MG cells might be responsible for the loss of cell polarity and inability to form lamellipodia, resulting in an overall inhibition of DK-MG cell migration (Figure 5C).

Judging from the results of both migration assays (Figures 1 and 2), the Hsp90 inhibitor AUY922 seems to be a more potent anti-migratory agent in GBM cells than the PI3K/mTOR inhibitor PI-103. The advantage of AUY922 is that, unlike PI-103 which had no effect on SNB19 cell migration, AUY922 strongly impeded the migration of both tested cell lines (Figures 1 and 2), independent of their mutation status in PTEN and $p 53$. As outlined in Supplementary Figure 6, the inhibition of cell migration by AUY922 seems to be mediated through various Hsp90 client proteins [15] such as AKT and MEK, which belong to different signaling pathways involved in cell motility and migration. On the cellular level, the AUY922-mediated alterations in the signaling pathways of SNB19 cells (Figure 4) were associated with massive changes in cell morphology and remodeling of the cytoskeleton. Particularly, in agreement with our results reported earlier [24], AUY922-treated SNB19 cells did not form multiple lamellipodia typical for control cells.

In conclusion, our study provides a proof-ofconcept that dual inhibition of PI3K/mTOR is a promising therapeutic strategy for preventing migration of GBM cells. In case of migrating cells that are not responsive to prolonged PI3K/mTOR inhibition due to an aberrant reactivation of the PI3K pathway, the therapeutic window needs to be carefully defined, or a combination of AKT and Hsp90 inhibitors should be considered.

\section{MATERIALS AND METHODS}

\section{Cell culture}

Human glioblastoma (GBM) cell lines, DK-MG and SNB19, were obtained from DSMZ (Braunschweig, Germany) and routinely cultured in complete growth medium (CGM) under standard conditions $\left(5 \% \mathrm{CO}_{2}\right.$, $\left.37^{\circ} \mathrm{C}\right)$. CGM contained Dulbecco's modified Eagle's medium (DMEM, Sigma, Deisenhofen, Germany) supplemented with $10 \%$ fetal bovine serum. The population doubling times for DK-MG and SNB19 cells were found to be about 48 and $25 \mathrm{~h}$, respectively. Both cell lines were authenticated by the supplier, used at low passage number $(<15)$ and were mycoplasma free (MycoAlert; Lonza, Rockland, ME).

\section{Drug treatment}

Both drugs were obtained from Selleckchem (Absource Diagnostics GmbH, Munich, Germany). The drugs were freshly diluted from frozen aliquots in DMSO stored at $-20^{\circ} \mathrm{C}$. PI-103 (2 $\mu \mathrm{M}$, [46]) and NVPAUY922 $(50 \mathrm{nM})$ were added $3 \mathrm{~h}$ prior to exposure to ionizing radiation (IR) and remained in CGM up to $24 \mathrm{~h}$ post-IR. Cells treated in parallel with respective amounts of DMSO served as controls. In order to allow the cells to reach confluency and to avoid cell detachment in the wound healing test, the formerly used NVP-AUY922 concentration of $200 \mathrm{nM}$ [47] was decreased to $50 \mathrm{nM}$. This concentration was used throughout the whole study.

\section{Antibodies}

The primary and secondary antibodies used in this study are specified in Supplementary Information.

\section{X-ray irradiation}

Irradiation was performed at room temperature using a 6 MV Siemens linear accelerator (Siemens, Concord, CA) at a dose rate of $2 \mathrm{~Gy} / \mathrm{min}$. After irradiation, cells were kept in CGM for the indicated time until harvest. 


\section{Time-lapse phase-contrast microscopy}

A Nikon BioStation IM-Q (Nikon, Melville, NY), which includes a cell incubator $\left(37^{\circ} \mathrm{C}, 5 \% \mathrm{CO}_{2}\right)$, a motorized inverted microscope and a CCD camera, was used to image live GBM cells over time by phase contrast microscopy. Prior to single-cell tracking experiments, about $10^{4}$ cells were plated into a Petri dish (diameter 35 $\mathrm{mm}$ ) containing $2 \mathrm{ml} \mathrm{CGM}$. In each experiment, timelapse images of several fields of view $(706 \times 530 \mu \mathrm{m})$ were acquired every $10 \mathrm{~min}$ over a 24 -h period, using a $\times 10$ phase contrast objective.

\section{Cell tracking and migration data analysis}

Cell tracking was performed using the software Time Lapse Analyzer (TLA; University of Ulm, Germany), which enables an automated cell identification and tracking procedure suitable for the evaluation of video sequences of unstained cells. The image preprocessing, segmentation, i.e. the separation of the objects of interest (cells) from the background, and cell tracking were performed as described elsewhere [48] with minor modifications. Briefly, the acquired digital images were processed with TLA software by using a combination of entropic and median filtering algorithms, which yielded two sets of binary images. By applying an AND gate to the data sets, individual cells were discriminated from the background and the coordinates of the centroids of cell nuclei were computed. Using this data, the cell trajectories were reconstructed using a modified nearestneighbor algorithm. Typically, 20 to 40 individual cells were tracked during each 24-h migration experiment. At least three experiments were conducted for each condition examined in the present study. From the acquired migration paths, cell migration speed and directionality of migration were calculated for each individual cell. Migration speed was defined as the total distance (TD) a cell travelled divided by the total time. Directionality (or persistence) of migration was defined as the net distance (ND, or displacement) from the starting position divided by the length of the total distance (TD), e.g. directionality $=1.0$ for migration in a straight line, directionality $=0.0$ for circular migration.

\section{Wound healing assay}

Wound healing in the presence or absence of inhibitors, with or without IR treatment, was analyzed directly and $18 \mathrm{~h}$ after scratching as previously described [24].

\section{Fluorescence staining of F-actin and p-FAK(Tyr397)}

Cells were cultured on glass slides to subconfluency, permeabilized and fixated as described previously [49]. Staining of F-actin was performed with a phalloidin-Dy647-conjugate. Immunostaining of p-FAK(Tyr397) was performed by incubating samples with a primary and respective secondary antibody (see Supplementary Materials), each for $2 \mathrm{~h}$ at room temperature. Confocal fluorescence images were acquired with a Zeiss LSM 700 microscope.

\section{dSTORM (direct stochastic optical reconstruction microscopy)}

The experimental setup for $d$ STORM was described previously in detail [23]. The main advantage of direct stochastic optical reconstruction microscopy ( $d$ STORM, [23]) over the conventional LSM is its superior spatial resolution of about $20 \mathrm{~nm}$ whereas in a standard LSM the resolution is limited to $\sim 250 \mathrm{~nm}$ due to light diffraction. $d$ STORM uses photoswitchable fluorescent dyes, which can be transferred to a reversible dark state with a lifetime ranging between $\sim 100 \mathrm{~ms}$ and a few seconds. A sparse subset of fluorophores is then spontaneously (stochastically) reactivated, allowing their precise localization. Photoswitching is based on thiol-mediated reduction of fluorescent dyes to a non-fluorescent dark state after excitation. Repetitive cycles of activation, localization and deactivation enable a temporal separation of fluorophores from which a spatially super-resolved image can be reconstructed. Prior to the measurements, $5 \times 10^{4}$ cells were seeded on 8 -well II chambered cover glasses (Sarstaedt, Nümbrecht, Germany). For data processing and image reconstruction, the open access software for single-molecule-based localization microscopy rapidSTORM 3.2 was used as previously described [50].

\section{Western blot}

For immunoblot analysis, whole-cell lysates were prepared $3 \mathrm{~h}$ and $24 \mathrm{~h}$ after addition of the drugs according to standard procedures. Samples equivalent to $20-40 \mu \mathrm{g}$ of protein were separated using 4-12\% SDS-polyacrylamide pre-cast gels (Invitrogen, Karlsruhe, Germany) and transferred to nitrocellulose membranes, as described previously [46].

\section{Statististics}

Data are presented as mean $\pm \mathrm{SE}$, unless otherwise noted. A Student's unpaired $t$-test was performed when statistical comparisons were made between two sets of data. The threshold of statistical significance was set at $P$-value of $<0.05$. Statistics was performed with Origin 8.5 (Microcal, Northampton, MA).

\section{CONFLICTS OF INTEREST}

The authors declare no conflicts of interest. 


\section{REFERENCES}

1. Stupp R, Mason WP, van den Bent MJ, Weller M, Fisher B, Taphoorn MJ, Belanger K, Brandes AA, Marosi C, Bogdahn U, Curschmann J, Janzer RC, Ludwin SK, et al. Radiotherapy plus concomitant and adjuvant temozolomide for glioblastoma. N Engl J Med. 2005; 352: 987-996.

2. Ulrich TA, de Juan Pardo EM, Kumar S. The mechanical rigidity of the extracellular matrix regulates the structure, motility, and proliferation of glioma cells. Cancer Res. 2009; 69: 4167-4174.

3. Reardon DA, Nabors LB, Stupp R, Mikkelsen T. Cilengitide: an integrin-targeting arginine-glycine-aspartic acid peptide with promising activity for glioblastoma multiforme. Expert Opin Investig Drugs. 2008; 17: $1225-1235$.

4. Maher EA, Furnari FB, Bachoo RM, Rowitch DH, Louis DN, Cavenee WK, DePinho RA. Malignant glioma: genetics and biology of a grave matter. Genes Dev. 2001; 15: 1311-1333.

5. Cancer Genome Atlas Research Network. Comprehensive genomic characterization defines human glioblastoma genes and core pathways. Nature. 2008; 455: 1061-1068.

6. Masui K, Cloughesy TF, Mischel PS. Review: molecular pathology in adult high-grade gliomas: from molecular diagnostics to target therapies. Neuropathol Appl Neurobiol. 2012; 38: 271-291.

7. Tamura M, Gu J, Matsumoto K, Aota S, Parsons R, Yamada KM. Inhibition of cell migration, spreading, and focal adhesions by tumor suppressor PTEN. Science. 1998; 280: 1614-1617.

8. Zhang S, Yu D. PI(3)king apart PTEN's role in cancer. Clin Cancer Res. 2010; 16: 4325-4330.

9. Martin S, Janouskova H, Dontenwill M. Integrins and p53 pathways in glioblastoma resistance to temozolomide. Front Oncol. 2012; 2: 157.

10. Muller PAJ, Vousden KH, Norman JC. p53 and its mutants in tumor cell migration and invasion. J Cell Biol. 2011; 192: 209-218.

11. Mak AS. p53 in cell invasion, podosomes, and invadopodia. Cell Adh Migr. 2014; 8: 205-214.

12. Gadea G, Roger L, Anguille C, de Toledo M, Gire V, Roux P. TNFalpha induces sequential activation of $\mathrm{Cdc} 42-$ and p38/p53-dependent pathways that antagonistically regulate filopodia formation. J Cell Sci. 2004; 117: 6355-6364.

13. Roger L, Gadea G, Roux P. Control of cell migration: a tumour suppressor function for p53? Biol Cell. 2006; 98: 141-152.

14. Zhou H, Huang S. Role of mTOR signaling in tumor cell motility, invasion and metastasis. Curr Protein Pept Sci. 2011; 12: 30-42.

15. Picard D. Heat-shock protein 90, a chaperone for folding and regulation. Cell Mol Life Sci. 2002; 59: 1640-1648.
16. Hayakawa M, Kaizawa H, Moritomo H, Koizumi T, Ohishi T, Okada M, Ohta M, Tsukamoto S, Parker P, Workman $\mathrm{P}$, Waterfield M. Synthesis and biological evaluation of 4-morpholino-2-phenylquinazolines and related derivatives as novel PI3 kinase p110alpha inhibitors. Bioorg Med Chem. 2006; 14: 6847-6858.

17. Raynaud FI, Eccles S, Clarke PA, Hayes A, Nutley B, Alix S, Henley A, Di-Stefano F, Ahmad Z, Guillard S, Bjerke LM, Kelland L, Valenti M, et al. Pharmacologic characterization of a potent inhibitor of class I phosphatidylinositide 3-kinases. Cancer Res. 2007; 67: 5840-5850.

18. Brough PA, Aherne W, Barril X, Borgognoni J, Boxall K, Cansfield JE, Cheung KM, Collins I, Davies NG, Drysdale MJ, Dymock B, Eccles SA, Finch H, et al. 4,5-diarylisoxazole Hsp90 chaperone inhibitors: potential therapeutic agents for the treatment of cancer. J Med Chem. 2008; 51: 196-218.

19. Jensen MR, Schoepfer J, Radimerski T, Massey A, Guy CT, Brueggen J, Quadt C, Buckler A, Cozens R, Drysdale MJ, Garcia-Echeverria C, Chène P. NVP-AUY922: a small molecule HSP90 inhibitor with potent antitumor activity in preclinical breast cancer models. Breast Cancer Res. 2008; 10: R33.

20. Gupta M, Doss B, Lim CT, Voituriez R, Ladoux B. Single cell rigidity sensing: a complex relationship between focal adhesion dynamics and large-scale actin cytoskeleton remodeling. Cell Adh Migr. 2016; 10: 554-567.

21. Maninová M, Vomastek T. Dorsal stress fibers, transverse actin arcs, and perinuclear actin fibers form an interconnected network that induces nuclear movement in polarizing fibroblasts. FEBS J. 2016; 283: 3676-3693.

22. Heilemann M, van de Linde S, Schüttpelz M, Kasper R, Seefeldt B, Mukherjee A, Tinnefeld P, Sauer M. Subdiffraction-resolution fluorescence imaging with conventional fluorescent probes. Angew Chem Int Ed Engl. 2008; 47: 6172-6176.

23. van de Linde S, Löschberger A, Klein T, Heidbreder M, Wolter S, Heilemann M, Sauer M. Direct stochastic optical reconstruction microscopy with standard fluorescent probes. Nat Protoc. 2011; 6: 991-1009.

24. Hartmann S, Günther N, Biehl M, Katzer A, Kuger S, Worschech E, Sukhorukov VL, Krohne G, Zimmermann H, Flentje M, Djuzenova CS. Hsp90 inhibition by NVPAUY922 and NVP-BEP800 decreases migration and invasion of irradiated normoxic and hypoxic tumor cell lines. Cancer Lett. 2013; 331: 200-210.

25. McCubrey JA, Steelman LS, Chappell WH, Abrams SL, Wong EW, Chang F, Lehmann B, Terrian DM, Milella M, Tafuri A, Stivala F, Libra M, Basecke J, et al. Roles of the Raf/MEK/ERK pathway in cell growth, malignant transformation and drug resistance. Biochim Biophys Acta. 2007; 1773: 1263-1284. 
26. Steelman LS, Chappell WH, Abrams SL, Kempf RC, Long J, Laidler P, Mijatovic S, Maksimovic-Ivanic D, Stivala F, Mazzarino MC, Donia M, Fagone P, Malaponte G, et al. Roles of the Raf/MEK/ERK and PI3K/PTEN/Akt/mTOR pathways in controlling growth and sensitivity to therapyimplications for cancer and aging. Aging (Albany NY). 2011; 3: 192-222. doi: 10.18632/aging.100296.

27. Huang C, Jacobson K, Schaller MD. MAP kinases and cell migration. J Cell Sci. 2004; 117: 4619-4628.

28. Taiyab A, Rao CM. HSP90 modulates actin dynamics: inhibition of HSP90 leads to decreased cell motility and impairs invasion. Biochim Biophys Acta. 2011; 1813: 213-221.

29. Amano M, Chihara K, Kimura K, Fukata Y, Nakamura N, Matsuura Y, Kaibuchi K. Formation of actin stress fibers and focal adhesions enhanced by Rho-kinase. Science. 1997; 275: 1308-1311.

30. Etienne-Manneville S, Hall A. Rho GTPases in cell biology. Nature. 2002; 420: 629-635.

31. Fukata M, Nakagawa M, Kaibuchi K. Roles of Rho-family GTPases in cell polarisation and directional migration. Curr Opin Cell Biol. 2003; 15: 590-597.

32. Qadir MI, Parveen A, Ali M. Cdc42: role in cancer management. Chem Biol Drug Des. 2015; 86: 432-439.

33. Di Cristofano A, Pandolfi PP. The multiple roles of PTEN in tumor suppression. Cell. 2000; 100: 387-390.

34. Memmel S, Sukhorukov VL, Höring M, Westerling K, Fiedler V, Katzer A, Krohne G, Flentje M, Djuzenova CS. Cell surface area and membrane folding in glioblastoma cell lines differing in PTEN and p53 status. PLoS One. 2014; 9: e87052.

35. Djuzenova CS, Fiedler V, Memmel S, Katzer A, Hartmann S, Krohne G, Zimmermann H, Scholz CJ, Polat B, Flentje M, Sukhorukov VL. Actin cytoskeleton organization, cell surface modification and invasion rate of 5 glioblastoma cell lines differing in PTEN and p53 status. Exp Cell Res. 2015; 330: 346-357.

36. Tinevez JY, Schulze U, Salbreux G, Roensch J, Joanny JF, Paluch E. Role of cortical tension in bleb growth. Proc Natl Acad Sci U S A. 2009; 106: 18581-18586.

37. Wild-Bode C, Weller M, Rimner A, Dichgans J, Wick W. Sublethal irradiation promotes migration and invasiveness of glioma cells: implications for radiotherapy of human glioblastoma. Cancer Res. 2001; 61: 2744-2750.

38. Arscott WT, Tandle AT, Zhao S, Shabason JE, Gordon IK, Schlaff CD, Zhang G, Tofilon PJ, Camphausen KA. Ionizing radiation and glioblastoma exosomes: implications in tumor biology and cell migration. Transl Oncol. 2013; 6: 638-648.

39. Choi YA, Lim HK, Kim JR, Lee CH, Kim YJ, Kang SS, Baek SH. Group IB secretory phospholipase A2 promotes matrix metalloproteinase-2-mediated cell migration via the phosphatidylinositol 3-kinase and Akt pathway. J Biol Chem. 2004; 279: 36579-36585.

40. Iijima M, Devreotes P. Tumor suppressor PTEN mediates sensing of chemoattractant gradients. Cell. 2002; 109: 599-610.

41. Samatar AA, Poulikakos PI. Targeting RAS-ERK signalling in cancer: promises and challenges. Nat Rev Drug Discov. 2014; 13: 928-942.

42. Mukhopadhyay UK, Mooney P, Jia L, Eves R, Raptis L, Mak AS. Doubles game: Src-Stat3 versus p53-PTEN in cellular migration and invasion. Mol Cell Biol. 2010; 30: 4980-4995.

43. Ströbele S, Schneider M, Schneele L, Siegelin MD, Nonnenmacher L, Zhou S, Karpel-Massler G, Westhoff MA, Halatsch ME, Debatin KM. A potential role for the inhibition of PI3K signaling in glioblastoma therapy. PLoS One. 2015; 10: e0131670.

44. Higuchi M, Masuyama N, Fukui Y, Suzuki A, Gotoh Y. Akt mediates Rac/Cdc42-regulated cell motility in growth factor-stimulated cells and in invasive PTEN knockout cells. Curr Biol. 2001; 11: 1958-1962.

45. Yamada KM, Araki M. Tumor suppressor PTEN: modulator of cell signaling, growth, migration and apoptosis. J Cell Sci. 2001; 114: 2375-2382.

46. Djuzenova CS, Fiedler V, Katzer A, Michel K, Deckert S, Zimmermann H, Sukhorukov VL, Flentje M. Dual PI3K- and mTOR-inhibitor PI-103 can either enhance or reduce the radiosensitizing effect of the Hsp90 inhibitor NVP-AUY922 in tumor cells: the role of drug-irradiation schedule. Oncotarget. 2016; 7:38191-38209. doi: 10.18632/ oncotarget.9501.

47. Stingl L, Stühmer T, Chatterjee M, Jensen MR, Flentje M, Djuzenova CS. Novel HSP90 inhibitors, NVP-AUY922 and NVP-BEP800, radiosensitise tumour cells through cell-cycle impairment, increased DNA damage and repair protraction. Br J Cancer. 2010; 102: 1578-1591.

48. Huth J, Buchholz M, Kraus JM, Schmucker M, von Wichert G, Krndija D, Seufferlein T, Gress TM, Kestler HA. Significantly improved precision of cell migration analysis in time-lapse video microscopy through use of a fully automated tracking system. BMC Cell Biol. 2010; 11: 24.

49. Andronic J, Shirakashi R, Pickel SU, Westerling KM, Klein T, Holm T, Sauer M, Sukhorukov VL. Hypotonic activation of the myo-inositol transporter SLC5A3 in HEK293 cells probed by cell volumetry, confocal and super-resolution microscopy. PLoS One. 2015; 10: e0119990.

50. Wolter S, Löschberger A, Holm T, Aufmkolk S, Dabauvalle MC, van de Linde S, Sauer M. rapidSTORM: accurate, fast open-source software for localization microscopy. Nat Methods. 2012; 9: 1040-1041. 